\title{
Real-time detection of implant-associated neutrophil responses using a formyl peptide receptor-targeting NIR nanoprobe
}

This article was published in the following Dove Press journal:

International Journal of Nanomedicine

19 April 2012

Number of times this article has been viewed

\section{Jun Zhou* \\ Yi-Ting Tsai* \\ Hong Weng \\ Ewin N Tang \\ Ashwin Nair \\ Digant P Davé \\ Liping Tang}

Department of Bioengineering, University of Texas at Arlington, Arlington, TX, USA

*Both authors contributed equally to this work
Correspondence: Liping Tang Department of Bioengineering, University of Texas at Arlington, PO Box 19138, Arlington, TX 76019-0138, USA

Tel +l 8172726075

Fax + I 8172722251

Email Itang@uta.edu

\begin{abstract}
Neutrophils play an important role in implant-mediated inflammation and infection. Unfortunately, current methods which monitor neutrophil activity, including enzyme measurements and histological evaluation, require many animals and cannot be used to accurately depict the dynamic cellular responses. To understand the neutrophil interactions around implant-mediated inflammation and infection it is critical to develop methods which can monitor in vivo cellular activity in real time. In this study, formyl peptide receptor (FPR)-targeting nearinfrared nanoprobes were fabricated. This was accomplished by conjugating near-infrared dye with specific peptides having a high affinity to the FPRs present on activated neutrophils. The ability of FPR-targeting nanoprobes to detect and quantify activated neutrophils was assessed both in vitro and in vivo. As expected, FPR-targeting nanoprobes preferentially accumulated on activated neutrophils in vitro. Following transplantation, FPR-targeting nanoprobes preferentially accumulated at the biomaterial implantation site. Equally important, a strong relationship was observed between the extent of fluorescence intensity in vivo and the number of recruited neutrophils at the implantation site. Furthermore, FPR-targeting nanoprobes may be used to detect and quantify the number of neutrophils responding to a catheter-associated infection. The results show that FPR-targeting nanoprobes may serve as a powerful tool to monitor and measure the extent of neutrophil responses to biomaterial implants in vivo.
\end{abstract}

Keywords: in vivo imaging, nanoprobe, neutrophils, inflammation, biocompatibility

\section{Introduction}

The accumulation of neutrophils in tissue is a hallmark of the acute inflammatory response. ${ }^{1-3}$ Following biomaterial implantation, many circulating neutrophils and macrophages/monocytes migrate from the blood stream to the implant's surrounding tissue. ${ }^{4,5}$ Subsequent interactions and cellular activation initiates the acute foreign body response. ${ }^{2,3}$ Studies have shown that recruited neutrophils release a large amount of granular enzymes and reactive oxygen species, including hydrogen peroxide and superoxide anions. ${ }^{6,7}$ Activated neutrophils and their products have also been shown to cause the degradation of polymeric implants and tissue engineering scaffolds. ${ }^{8,9}$ Furthermore, it has been suggested that the accumulation of activated neutrophils around biomaterial implants may lead to increased fibrotic reactions, delayed tissue regeneration, or even tissue damage. ${ }^{10,11}$ Moreover, the impaired bactericidal activities of implant-associated neutrophils are thought to be responsible for the pathogenesis of device-centered infection. ${ }^{12}$ Therefore, vast research efforts have been placed on studying the interactions between biomaterial implants and immune cell responses. 
It is well established that there is a good relationship between the number of recruited neutrophils and the extent of biomaterial-mediated inflammatory responses. ${ }^{4,5}$ The degree of the implant-associated neutrophil response may be quantified by either myeloperoxidase enzyme measurement ${ }^{4,5}$ or histological staining. ${ }^{13,14}$ However, these methods have at least three inherent disadvantages. First, to determine the kinetics of foreign body reactions, a large number of animals is needed to obtain sufficient tissue samples for studying the neutrophil interaction at various time points. ${ }^{15,16}$ Second, histological sectioning, staining, and tissue image analyses are very laborintensive and time-consuming. Finally, these conventional methods are cost-prohibitive for carrying out large scale studies required to develop a better understanding of the influence of material properties on foreign body reactions. To resolve these drawbacks, the overall goal of this investigation was to develop a fast, accurate, and simple method to assess neutrophil responses to biomaterial implants.

Several imaging methods have been developed to monitor the migration of neutrophils. Specifically, ${ }^{11}$ indium-labeled neutrophils and ${ }^{99 \mathrm{~m}}$ technetium-labeled IL-18BP-Fc-IL-1 ra (interleukin-18 binding protein-Fc-interleukin-1 receptor antagonist) recombinant human fusion protein uptakes have been shown to detect neutrophil responses. ${ }^{17}$ Two-photon microscopy and time-lapse imaging have also been used to study neutrophil trafficking in the lungs of mechanically ventilated mice. ${ }^{18}$ Several chemotactic peptide receptor agonists have also been investigated for their use in imaging infection and associated inflammatory cell accumulation in vivo. ${ }^{19,20}$ A leukotriene B4 receptor antagonist, ${ }^{99 \mathrm{~m}}$ technetium-RP517, has exhibited a neutrophil targeting ability and has been developed for imaging acute myocardium inflammation. ${ }^{21}$ Recently, optical imaging systems have been developed to target neutrophils. Specifically, studies have shown that the peptide cinnamoyl-Phe-(D)Leu-Phe-(D)Leu-Phe (cFLFLF) has a high affinity to the formyl peptide receptor (FPR) of neutrophils, ${ }^{22}$ and that near-infrared (NIR)-conjugated cFLFLF can be used to detect activated neutrophils in a mouse model of ear inflammation. ${ }^{23}$ However, the high hydrophobicity of cFLFLF often leads to a relatively poor target-to-background ratio for in vivo imaging. ${ }^{22}$ To address this deficiency, polyethylene glycol (PEG) polymers have been used to improve the hydrophilicity of the cFLFLF-NIR probes. These improved probes have enhanced efficacy and have been used to detect severe neutrophil-associated lung inflammation and infection. ${ }^{22-24}$ However, it is not clear whether a similar approach can be used to fabricate imaging probes for detecting and quantifying the degree of neutrophil recruitment related to medical device-associated foreign body reactions in vivo.

To find the answer, FPR-targeting nanoprobes were synthesized by crosslinking NIR dye into an eight-arm PEG platform. This complex was further conjugated with the FPR-targeting peptide, cFLFLF. The efficacy of FPRtargeting nanoprobes to recognize activated neutrophils was first studied in vitro. Using an in vivo imaging system, the effectiveness of FPR-targeting nanoprobes in quantifying the extent of biomaterial implant-mediated neutrophil recruitment was also assessed. Finally, FPR-targeting nanoprobes were also tested for their ability to continuously monitor and quantify the extent of the inflammatory response to different biomaterial implants and infected catheters in vivo.

\section{Material and methods Materials}

Eight-arm PEG-amine (40,000 molecular weight) was obtained from JenKem Technology USA Inc (Allen, TX). The peptide, cFLFLF, was custom synthesized by United BioSystems Inc (Rockville, MD) and an additional glutamic acid was added to the $\mathrm{C}$-terminus of the peptide for permitting PEG polymer conjugation (ie, cFLFLF-COOH). Oyster ${ }^{\circledR}-800$ TFP Ester was purchased from Boca Scientific Inc (Boca Raton, FL). 1-Ethyl-3-(3-(dimethylaminopropyl)carbodiimide was purchased from Thermo Scientific Pierce Protein Research Products (Rockford, IL). N-hydroxysuccinimide and dimethyl sulfoxide were purchased from Sigma-Aldrich Corporation (St Louis, MO). All other chemicals were purchased from Sigma-Aldrich.

\section{Preparation and characterization of the FPR-targeting nanoprobe}

FPR-targeting nanoprobes were prepared by incubating eight-arm PEG-amine $(20 \mathrm{mg} / 1.0 \mathrm{~mL}$ phosphate buffer, $50 \mathrm{mM}, \mathrm{pH} 7.4)$ with Oyster- $800(2.0 \mathrm{mg} / 1.0 \mathrm{~mL}$ phosphate buffer). After incubation at room temperature for 4 hours, the eight-arm PEG-Oyster-800 was dialyzed and then lyophilized. The peptide cFLFLF $(8.3 \mathrm{mg})$ was dissolved in $1.0 \mathrm{~mL}$ dimethyl sulfoxide in the presence of $4.5 \mathrm{mg}$ 1-Ethyl-3-(3-(dimethylaminopropyl)carbodiimide and $4.5 \mathrm{mg} \mathrm{N}$-hydroxysuccinimide to activate the carboxyl group $(-\mathrm{COOH})$ at the $\mathrm{C}$-terminus of the peptides. Following overnight incubation at $4{ }^{\circ} \mathrm{C}$, the activated peptide solution was mixed with eight-arm PEG-Oyster-800 solution (peptide:eight-arm PEG-Oyster-800=27:1 molar ratio). The mixtures were incubated at room temperature for 24 hours prior to dialysis and lyophilization. The FPR-targeting 
nanoprobe (cFLFLF-PEG-Oyster-800, $1.0 \mathrm{mg} / \mathrm{mL}$ ) was dissolved in sterilized distilled water and the optical property of the nanoprobe was measured using a fluorescence spectrometer (RF-5301PC; Shimadzu Corporation, Tokyo, Japan), as described previously. ${ }^{25}$ The peptide to dye conjugation ratio was determined using an ultravioletvisible spectrometer (Lambda 19 Spectrometer; PerkinElmer, Waltham, MA) according to the manufacturer's instruction and a previous publication. ${ }^{26}$ Briefly, with a known amount of nanoprobes, the relative dye and peptide concentrations were determined using the spectrometer. Measurements were performed at $778 \mathrm{~nm}$ and $280 \mathrm{~nm}$ wavelengths for the dye and peptide, respectively; the extinction coefficient was provided by the manufacturer. Values were then compared to a standard concentration curve. The average value of peptide and dye per nanoprobe was calculated by dividing the respective concentrations by the total amount of the nanoprobe.

\section{In vitro neutrophil homing of the FPR-targeting nanoprobe}

To assess the ability of the FPR-targeting nanoprobe to detect activated neutrophils, activated mouse neutrophils $\left(9 \times 10^{6}\right.$ cells $/ \mathrm{mL}$ ) isolated via peritoneal lavage from BALB/c mice were used, as described previously., ${ }^{5,27}$ Various numbers of neutrophils were seeded into each well of a 96-well plate prior to incubating with either the FPR-targeting nanoprobe or its control (PEG-Oyster-800) $(40 \mu \mathrm{L}$ at $0.4 \mathrm{mg} / \mathrm{mL})$ at $37^{\circ} \mathrm{C}$ for 30 minutes. At the end of the study, each well was washed three times with phosphate buffered saline ( $50 \mathrm{mM}, \mathrm{pH} 7.4)$ to remove the unbound nanoprobes. Neutrophil-associated fluorescence intensities were then recorded using a microplate reader (Infinite ${ }^{\circledR}$ M200; Tecan Group Ltd, Mannedorf, Switzerland) at an excitation wavelength of $760 \mathrm{~nm}$ and an emission wavelength of $830 \mathrm{~nm}$. All the experiments were conducted in triplicate. Similar studies were also carried out on glass slides for microscopic optical imaging. Following nanoprobe incubation, the adherent cells on glass slides were also incubated with the fluorescein isothiocyanate-conjugated antineutrophil antibody (ab2557; Abcam, Cambridge, MA) at $37^{\circ} \mathrm{C}$ for 30 minutes prior to being washed three times with phosphate buffered saline. The adherent cells were then observed under an Axiovert ${ }^{\circledR} 200$ microscope (Carl Zeiss MicroImaging, Thornwood, NY) with a Chroma Cy7TM filter cube (excitation filter HQ710/75× dichroic Q750LP BS, and emission filter HQ810/90 m; Chroma Technology Corporation, Bellows Falls, VT) and a Zeiss filter set 10 (excitation filter BP 450-490, dichroic mirror FT 510, and emission filter BP 515-565; Carl Zeiss). Cell images were captured with a CCD camera (AxioCam MRm), and image analysis was performed with AxioVision ${ }^{\circledR} 3.1$ imaging software (Carl Zeiss).

\section{Inflammation and infection animal model}

$\mathrm{BALB} / \mathrm{c}$ mice (female, 20-25 gram body weight) were purchased from Taconic Farms Inc (Germantown, NY) and were used for all in vivo studies. The animal protocols were approved by the University of Texas at Arlington's Animal Care and Use Committee. To induce localized inflammatory responses, lipopolysaccharide (LPS; $100 \mu \mathrm{g} / 50 \mu \mathrm{L}$ saline) or saline (as control) was injected subcutaneously on the dorsal area of mice. Poly(lactic acid) (PLA) microparticles and PEG nanoparticles were used as control subjects to trigger various extents of foreign body reactions. PLA microparticles (5-10 $\mu \mathrm{m}$ in diameter) and PEG nanoparticles (100 nm in diameter) were synthesized as previously demonstrated. ${ }^{28,29}$ As recently described, $100 \mu \mathrm{L}$ of the particles or saline (as control) were injected into the subcutaneous space. ${ }^{25}$ Limited experiments were also carried out using neutrophil-depleted mice. Neutrophil depletion was performed based on a published protocol..$^{30}$ In brief, animals were injected intraperitoneally with $100 \mu \mathrm{L}$ of neutrophil neutralizing antibody (rabbit anti-mouse neutrophil antibody; Accurate Chemical and Scientific Corporation, Westbury, NY) at 18 hours and additionally at 1 hour prior to the experiments. To study indwelling catheter-associated inflammatory responses and infection, polyurethane (PU) catheters (1 cm in length) obtained from Sentry Medical Products (Green Bay, WI) were incubated with saline or luciferase-transgene Staphylococcus aureus Xen29 strain $\left(1.6 \times 10^{8}\right.$ colony-forming units $/ \mathrm{mL}$; Caliper Life Sciences, Hopkinton, MA) at $37^{\circ} \mathrm{C}$ for 3 hours and then implanted subcutaneously in mice following an established protocol. ${ }^{31-33} \mathrm{After}$ the implantation of particles, saline, or catheters for 24 hours, animals were intravenously administered with $60 \mu \mathrm{L}$ of the FPR-targeting nanoprobe $(0.4 \mathrm{mg} / \mathrm{mL}) 3$ hours prior to imaging analyses.

\section{Imaging analyses of the whole body and harvested organs}

The whole body fluorescence images were taken using the In-Vivo FX Pro system (f-stop: 2.5, excitation filter: $760 \mathrm{~nm}$, emission filter: $830 \mathrm{~nm}, 4 \times 4$ binning; Carestream Health, Rochester, NY). For imaging analyses, regions of interest were drawn over the implantation locations in the fluorescence images, and the mean intensities for all pixels in the regions of interest were calculated. All data analyses were performed with the Carestream Molecular Imaging Software, 
Network Edition 4.5 (Carestream Health). To assess the biodistribution of FPR-targeting nanoprobes in vivo, animals were sacrificed and tissues were rapidly dissected. The isolated organs/tissues were then immediately imaged using the In-Vivo FX Pro system.

\section{Histological analysis of localized inflammatory responses}

To assess the extent of neutrophil responses in various models, the implants and surrounding tissue were isolated for histological evaluation as described earlier. ${ }^{34,35}$ Hematoxylin and eosin staining was performed on all samples to assess the overall inflammatory reactions. To quantify the number of recruited neutrophils, some tissue sections were immunohistochemically stained with pan-neutrophil antibody (Santa Cruz Biotechnology, Santa Cruz, CA) and then with peroxidase-conjugated goat anti-rat secondary antibodies (Jackson ImmunoResearch Laboratories, West Grove, PA). All histological imaging analyses were performed on a Leica microscope (Leica Microsystems $\mathrm{GmbH}$, Wetzlar, Germany) and processed using $\mathrm{NIH}$ ImageJ (National Institutes of Health, Bethesda, MD). ${ }^{34}$ To visualize the distribution of the FPR-targeting nanoprobe in inflamed tissues, fresh tissue sections were also imaged using an Axiovert 200 microscope with an NIR filter cube as described previously. ${ }^{25}$

\section{Statistical analysis}

The statistical comparison between different treatment groups was carried out using Student's $t$-test. Differences were considered statistically significant when $P \leq 0.05$. Linear regression analyses and the correlation coefficient were calculated to reflect the relationship between fluorescence intensities and neutrophil numbers in vitro and in vivo.

\section{Results}

\section{Characterization of the FPR-targeting nanoprobe}

PEG has been widely used as a polymeric carrier in polymerbased drug delivery and as an imaging probe due to its low toxicity, low nonspecific binding, and prolonged blood circulation time. ${ }^{36}$ In this study, the FPR-targeting nanoprobe was prepared by sequentially conjugating NIR dye and the peptide cFLFLF into an amino-terminated eight-arm PEG platform through 1-Ethyl-3-(3-(dimethylaminopropyl)carbodiimide coupling chemistry (Figure 1A). ${ }^{37,38}$ Ultraviolet-visible spectrometer measurements show that unlike the control probes FPR-targeting nanoprobes have an absorbance peak at $280 \mathrm{~nm}$, identical to the peak absorbance wavelength of the peptide (Figure 1B). These results support the conclusion of successful conjugation of peptide ligands to FPRtargeting nanoprobes. On average, each mole of nanoprobe was found to contain 1.8 moles of dye and 6.0 moles of peptide. Furthermore, fluorescence spectroscopic results demonstrated that the conjugation of NIR dye did not significantly alter the fluorescence spectra of free Oyster-800 dye with a maximum emission at $799 \mathrm{~nm}$ and excited at 785 nm (Figure 1C).

\section{FPR-targeting nanoprobes recognize activated neutrophils in vitro}

To examine their ability to recognize activated neutrophils in vitro, FPR-targeting nanoprobes (NIR-PEG-cFLFLF) or control probes (NIR-PEG) were incubated with mouse neutrophils. The cells were also stained with a rat antimouse monoclonal neutrophil antibody and a fluorescein isothiocyanate-conjugated goat anti-rat secondary antibody for neutrophil confirmation. The cells were then observed using an optical microscope. As anticipated, it was found that only activated neutrophils (stained with the antibody, green color) were associated with the FPR-targeting nanoprobe (red color) (Figure 1D). This finding supports the hypothesis that FPR-targeting nanoprobes may be used to specifically identify activated neutrophils. To further study whether the nanoprobes can be used to quantify the number of activated neutrophils in vitro, various concentrations of neutrophils were incubated with the probes and the neutrophil-associated fluorescence intensities were determined. In support of the hypothesis, it was found that there was a linear correlation between the fluorescence intensity and the number of neutrophils (Figure 1E). In contrast, the fluorescence intensity of neutrophils incubated with the control nanoprobes (NIR-PEG) remained close to the background intensity regardless of the cell number. Overall, the in vitro results support that FPR-targeting nanoprobes can preferentially bind to neutrophils and may be used to quantify the number of neutrophils in vitro.

\section{Detection of LPS-induced localized neutrophil responses using FPR-targeting nanoprobes}

The effectiveness of FPR-targeting nanoprobes for detecting neutrophil accumulation in inflamed tissue was first tested in vivo using an LPS-induced inflammatory model. After LPS 
A

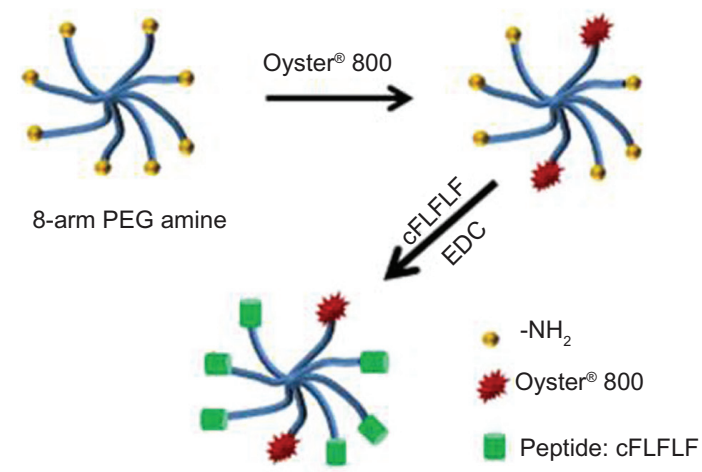

C

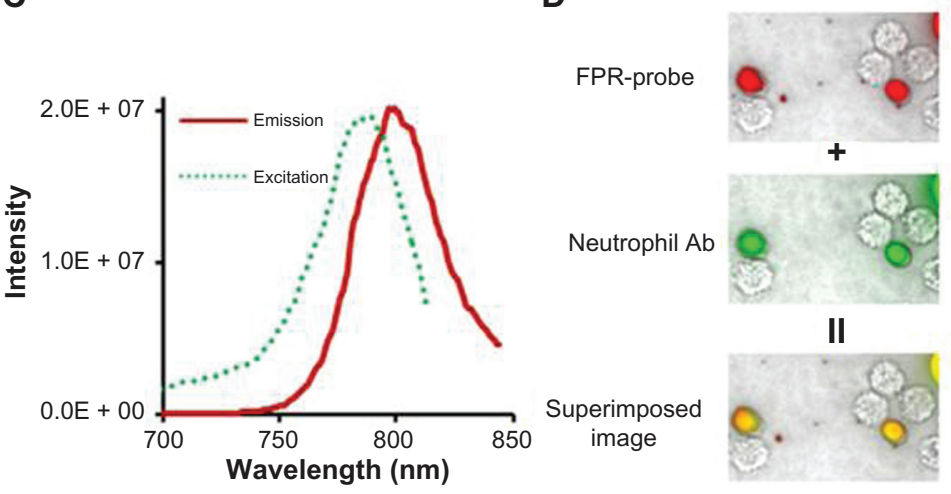

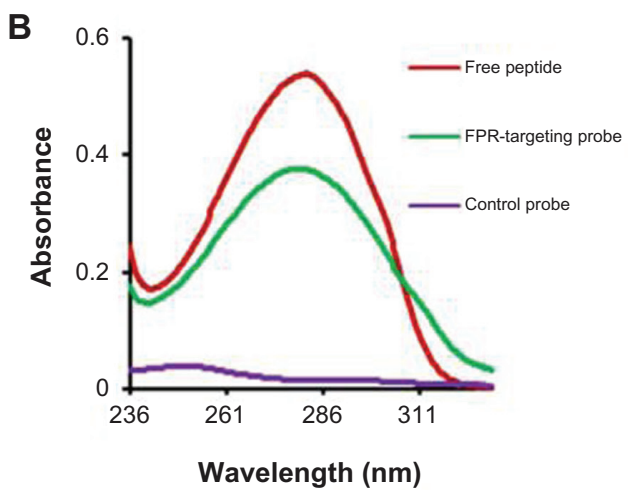

E

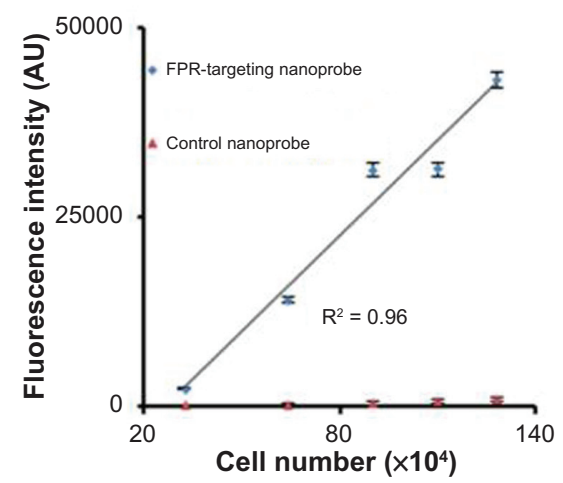

Figure I Fabrication and characterization of formyl peptide receptor-targeting nanoprobes and in vitro study to assess the specificity of formyl peptide receptor-targeting nanoprobes to activated neutrophils. (A) Schematic illustration of formyl peptide receptor-targeting nanoprobes. (B) Absorbance measurements of peptides, formyl peptide receptor-targeting nanoprobes, and control nanoprobes. (C) Excitation and emission spectra of formyl peptide receptor-targeting nanoprobe. (D) Fluorescence microscopy images of activated neutrophils incubated with formyl peptide receptor-targeting nanoprobe (red color) and neutrophil-specific antibody (green color) and their superimposed image. (E) Correlation between neutrophil numbers and neutrophil-associated fluorescence intensities following incubation with either formyl peptide receptor-targeting nanoprobe or control probe.

Abbreviations: cFLFLF, cinnamoyl-Phe-(D)Leu-Phe-(D)Leu-Phe; EDC, I-Ethyl-3-(3-(dimethylaminopropyl)carbodiimide; FPR, formyl peptide receptor; NH ${ }_{2}$, amine; PEG, polyethylene glycol; $\mathrm{R}^{2}$, correlation coefficient.

subcutaneous administration for 24 hours, the FPR-targeting nanoprobe was injected intravenously and whole body images were recorded at different time points (Figure 2A). A substantial increase in fluorescence intensity was found at the LPS injection site, in as short as 30 minutes following nanoprobe injection, in comparison to the control. The preferential accumulation of the FPR-targeting nanoprobe at the LPS site increased with time and reached its plateau at 2-3 hours, where the LPS treatment triggered 8.6 times higher fluorescence intensity than the control. To determine the biodistribution of the nanoprobes, the fluorescence images of the isolated organs or tissues were recorded and used to quantify the organ/tissue-associated fluorescence intensities (Figure 2B). As anticipated, large quantities of the FPR-targeting nanoprobe were found in the liver and kidney. A substantial amount of fluorescence from the nanoprobes was also found in the lung, heart, and pancreas. In agreement with the whole body imaging results, it was found that the average fluorescence intensity at the LPS injection sites was
4.5 times higher than the control tissues (saline injection) (Figure 2B). Based on the in vitro results, the authors believe that the accumulation of FPR-targeting nanoprobes at the LPS injection site is likely associated with the accumulation of neutrophils in the tissue. Indeed, using hematoxylin and eosin staining and immunohistochemical staining, it was found that the LPS injection triggered an increase in inflammatory cells (ten times higher) and neutrophils (90 times higher) in comparison to the control tissue (Figure 2C).

\section{Use of FPR-targeting nanoprobes to assess neutrophil accumulation near PLA implants}

The results thus far support that the FPR-targeting nanoprobes can detect LPS-elicited severe inflammatory responses. It is not clear, however, whether the FPR-targeting nanoprobes can also determine mild or localized inflammatory responses, such as foreign body reactions and associated neutrophil reactions. To find the answer, mice were subcutaneously 
A

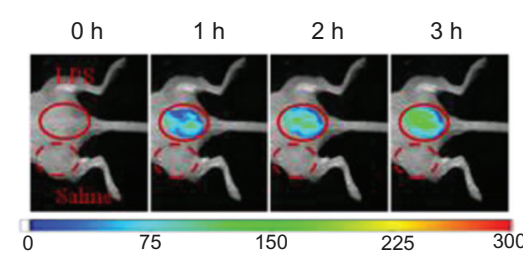

B
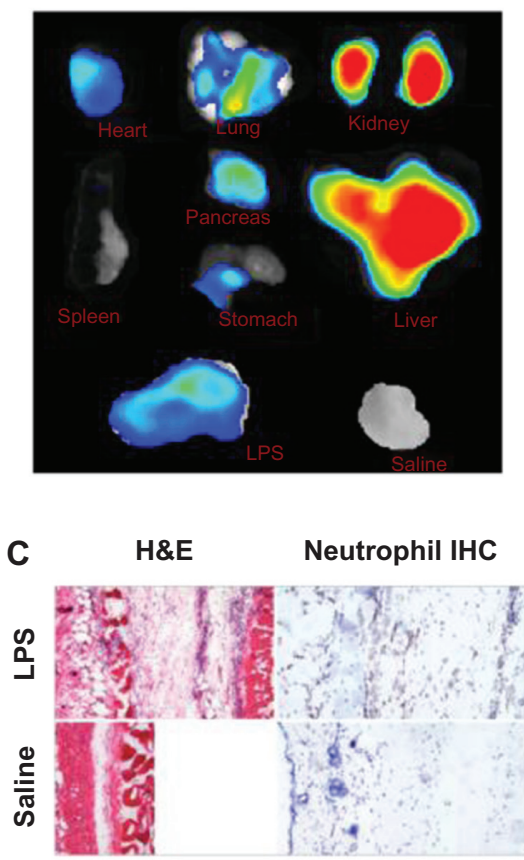
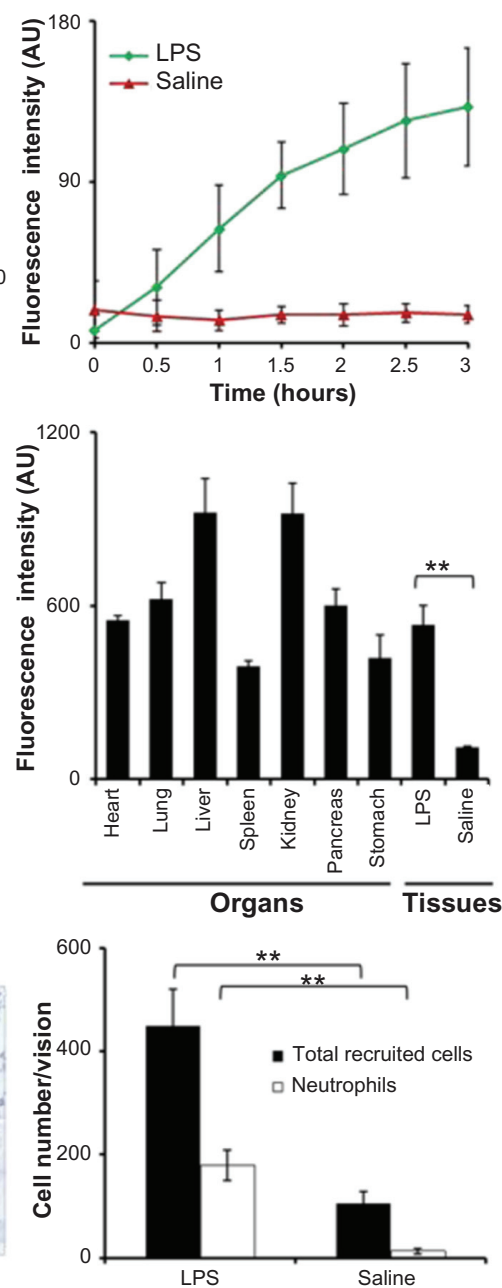

Figure 2 In the lipopolysaccharide-induced severe inflammation model, the formyl peptide receptor-targeting probe was administered intravenously 24 hours after a lipopolysaccharide subcutaneous injection. (A) The merged fluorescence and white light images (left panel) show the accumulation of the formyl peptide receptor-targeting probe at the lipopolysaccharide injection sites, but not the control site (saline injection). The mean fluorescence intensities at the lipopolysaccharide injection sites, but not saline injection sites, increased with time (right panel). (B) Ex vivo image (left) and the mean fluorescence intensity (right) of isolated tissues and organs show the overall probe biodistribution and also confirm the preferential accumulation of formyl peptide receptor-targeting probes in the inflamed tissue. (C) Representative hematoxylin and eosin staining (200x) and immunohistochemical neutrophil staining (400x) of the injection sites (left panel). Quantification of inflammatory cells (based on hematoxylin and eosin staining) and neutrophils (based on immunohistochemical staining) assured severe inflammatory responses and increased neutrophil accumulation at the lipopolysaccharide injection sites (right panel).

Abbreviations: H\&E, hematoxylin and eosin; IHC, immunohistochemical; LPS, lipopolysaccharide.

implanted with PLA microparticles for 24 hours to trigger foreign body reactions. The animals were subsequently administered intravenously with the FPR-targeting nanoprobes. As expected, PLA microparticle implantation triggered foreign body reactions which were accompanied by a large number of inflammatory cells and neutrophils (Figure 3A and B). The recruited neutrophils most likely play an essential role in the pathogenesis of the foreign body reactions. This is clearly observed through neutrophil depletion studies which show a substantial reduction in the extent of inflammatory cell $(\sim 50 \%)$ and neutrophil $(\sim 75 \%)$ recruitment to PLA implant sites when compared with controls (Figure $3 \mathrm{~A}$ and B). To validate that the nanoprobe accumulation is primarily due to the infiltration of neutrophils, both control and neutrophildepleted animals were subcutaneously implanted with PLA particles and then intravenously administered with the FPR-targeting nanoprobes. As expected, neutrophil depletion resulted in decreased fluorescence intensity from the PLA implant site at 1 hour ( $100 \%$ intensity reduction) and 4 hours ( $82 \%$ intensity reduction) in comparison to the control animals (Figure 3C). These findings demonstrate the high efficiency of FPR-targeting nanoprobes in recognizing and targeting activated neutrophils at the inflamed sites. Furthermore, it is likely that the nanoprobe can be used to detect implant-associated neutrophil accumulation and foreign body reactions. 
A

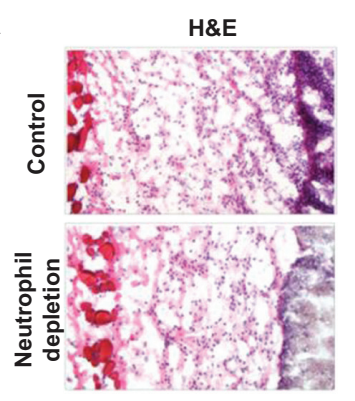

B
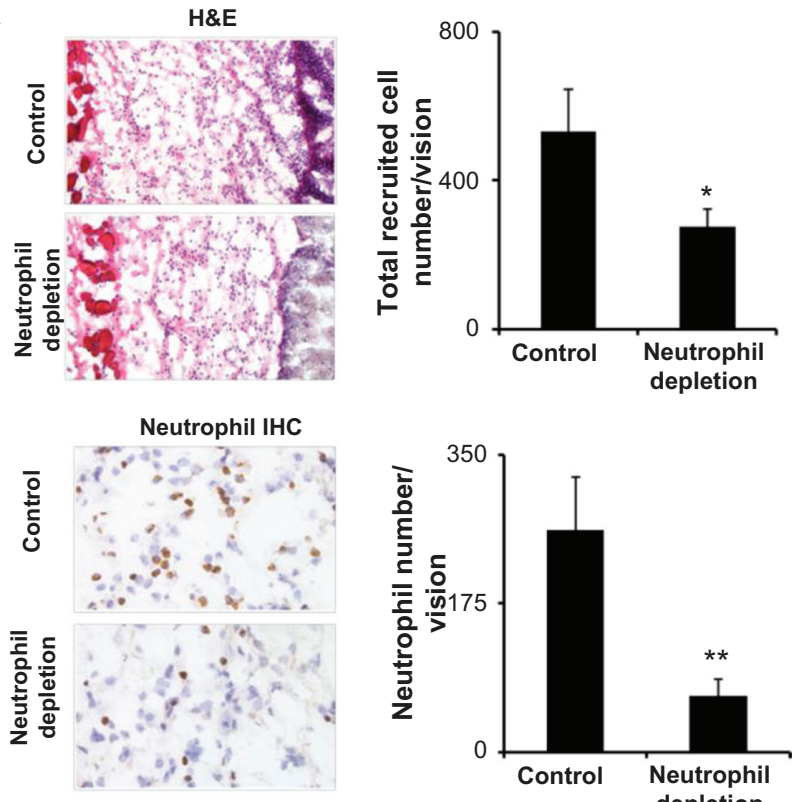

C

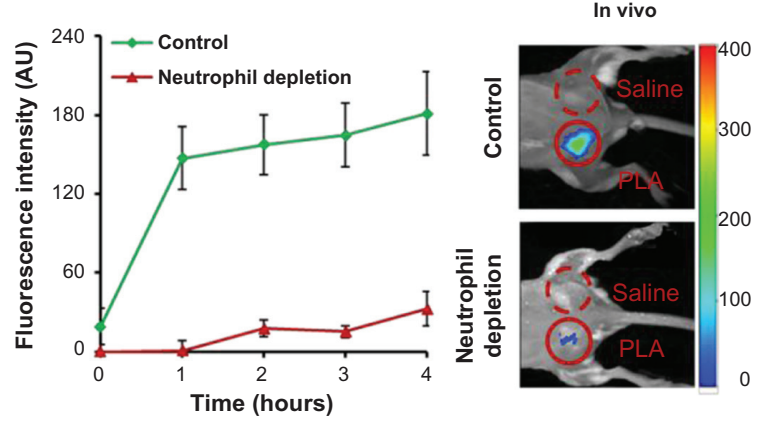

Figure 3 Poly(lactic acid) particles were implanted in neutrophil-depleted versus control mice for 24 hours prior to formyl peptide receptor-targeting nanoprobe administration. (A) Representative image of hematoxylin and eosin staining $(200 \times)$ of the implant sites and quantification analysis of inflammatory cells. (B) Representative image of immunohistochemical staining $(600 \times)$ and quantification analysis of neutrophils. (C) Fluorescence intensities at different time points (left panel) and in vivo image at 4 hours postinjection (right panel) illustrate the diminishing accumulation of formyl peptide receptor-targeting probes in neutrophildepleted animals in comparison with controls.

Abbreviations: H\&E, hematoxylin and eosin; IHC, immunohistochemical; PLA, poly(lactic acid).

\section{Quantify the degree of foreign body reactions using FPR-targeting nanoprobes}

It is well established that biomaterial implants trigger various extents of neutrophil responses. Additionally, there is a good relationship between neutrophil recruitment and biomaterial-mediated acute inflammatory responses. ${ }^{4,5}$ It may therefore be possible to use FPR-targeting nanoprobes to assess the extent of foreign body reactions and biomaterial tissue compatibility. To test this hypothesis, PLA and PEG particles were selected as model biomaterials. Previous studies have shown that PLA and PEG particles prompt mild and weak inflammatory responses, respectively. ${ }^{25,39}$
The particles were subcutaneously implanted in mice, with saline as a control, for 24 hours prior to administration of the imaging nanoprobe. Three hours after the probe injection, whole body imaging was performed. The results show that the fluorescence signal at the PLA and PEG implantation sites were much higher than at the control saline site (Figure 4A). Quantitative analysis shows that PLA and PEG trigger 9.5 and 2.8 times higher fluorescence intensity than the control, respectively. To determine the distribution of the nanoprobes within the implantation sites, tissue sections of the PLA and PEG implants were observed under a fluorescence microscope (Figure 4B). It was found that a high concentration of nanoprobes was present in tissue neighboring the PLA implants, while significantly lower amounts were detected surrounding the PEG implants. Histological analysis shows that PLA particles prompted a greater extent of neutrophil accumulation at the implant site than PEG implants, while negligible neutrophils populated the control site (Figure 4C). Quantitative analysis reveals that 2.5 times more neutrophils accumulated around the PLA particles than the PEG particles (Figure 4D). A comparison and correlation between fluorescence intensity and neutrophil counts was further performed to determine a potential relationship between the nanoprobe accumulation and implant-mediated neutrophil responses. The results show a near linear trend (correlation coefficient $=0.89$ ) (Figure 4E). These findings confirm that FPR-targeting nanoprobes can be employed as an effective imaging probe not only to mark the inflammation location but also to estimate the extent of biomaterial-mediated neutrophil infiltration and inflammatory responses in vivo.

\section{Detection of infected catheters by means of FPR-targeting nanoprobes}

Given the ability of the nanoprobe to preferentially target neutrophils associated with particle implants in vivo, it was further investigated whether the nanoprobe could be used to detect inflammatory responses elicited by bacteria-colonized medical devices. For that, PU catheters were used as a model medical device. Some of the PU catheters were inoculated with the luciferase transgenic $S$. aureus for 24 hours prior to subcutaneous implantation. The FPR-targeting nanoprobes were then intravenously administered and images were captured 3 hours after the nanoprobe injection. Strong bioluminescent signals from the infected catheters were found, but not from the control catheters, demonstrating the colonization of bacteria on PU catheters in vivo (Figure 5A). In addition, bright fluorescence signals were found at the area of the infected catheters (Figure 5B). Analysis and quantification 
A

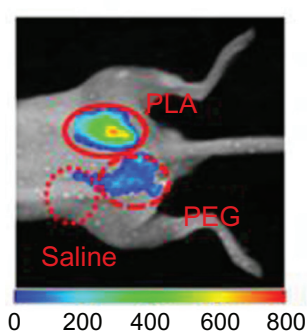

B

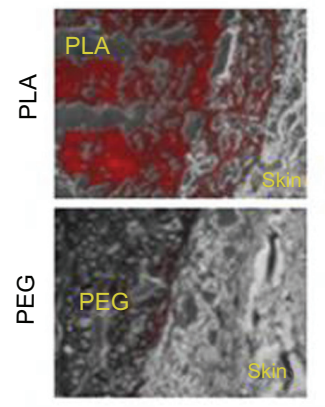

D

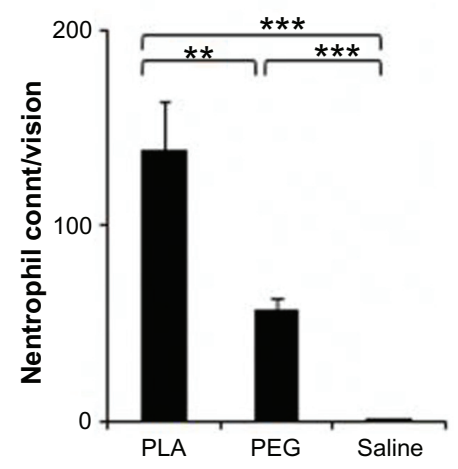

C
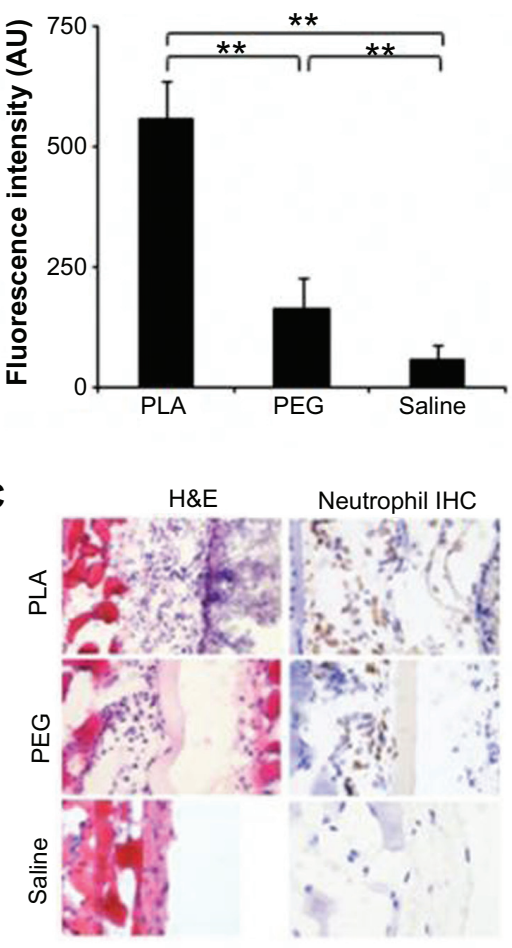

E

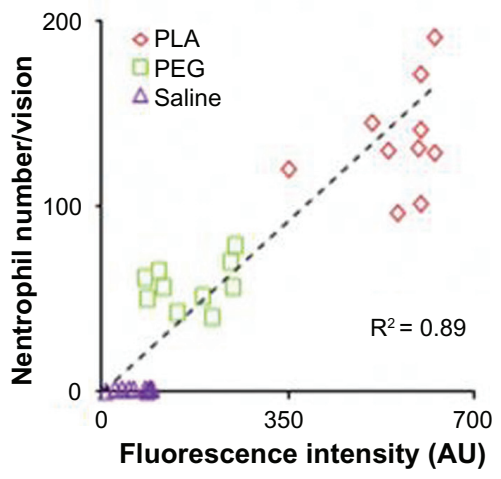

Figure 4 In a foreign body response animal model, poly(lactic acid) particles, polyethylene glycol particles, and saline were implanted subcutaneously in the back of animals for 24 hours prior to formyl peptide receptor-targeting probe injection. The animal images were taken 3 hours after probe administration. (A) Representative in vivo fluorescence imaging (left) and quantitative analysis (right) of the fluorescence intensity at different implantation sites (poly[lactic acid], polyethylene glycol, and saline). (B) Near-infrared fluorescence acquisition (red) superimposed onto the correlative phase contrast microscopy image (20x). The tissue section was unstained and unfixed to preserve the fluorescence signal. (C) Representative immunohistochemical staining of neutrophils (600x) and hematoxylin and eosin staining (400x) and (D) quantification of neutrophil numbers in tissue injected with poly(lactic acid), polyethylene glycol particles, or saline. (E) Correlation between recruited neutrophil numbers (based on histological analyses) and fluorescence intensities at the implant site.

Abbreviations: H\&E, hematoxylin and eosin; IHC, immunohistochemical; PEG, polyethylene glycol; PLA, poly(lactic acid); R², correlation coefficient.

of the fluorescence signal shows 8.8 times higher nanoprobe accumulation near the infected catheters versus the controls (Figure 5B). Further analysis was performed on tissue sections confirming a substantial increase in nanoprobe concentration in tissue surrounding the infected PU catheter in comparison to the controls (sterile PU catheter implants) (Figure 5C). Furthermore, histological analysis confirmed that the neutrophil population surrounding the infected tissue was 7.3 times higher than the tissue surrounding the control PU catheters (Figure 5D and E). These results suggest that
FPR-targeting nanoprobes can be used to monitor neutrophil responses to catheter implants. In addition, the nanoprobe is able to distinguish infected catheters versus sterile catheters, based on the extent of neutrophil responses.

\section{Discussion}

The development of imaging nanoprobes is essential to monitoring foreign body reactions. In the current study, the peptide cFLFLF was selected as a targeting ligand based on the following advantages. First, several recent studies 
A

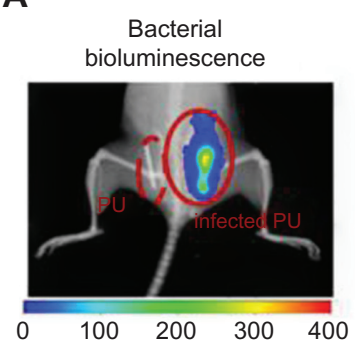

Bacterial number

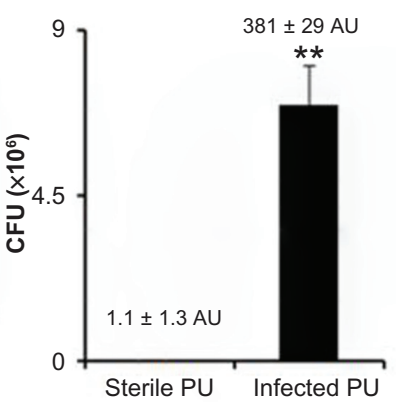

B

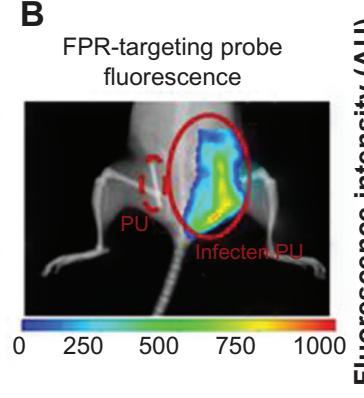

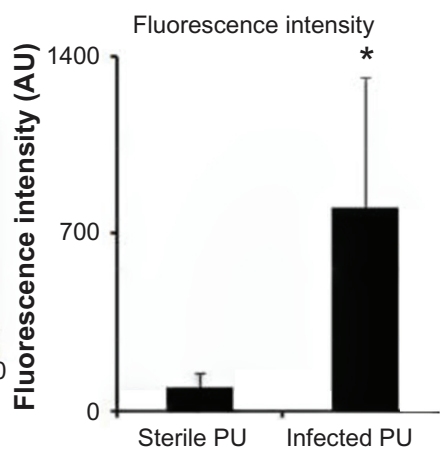

C
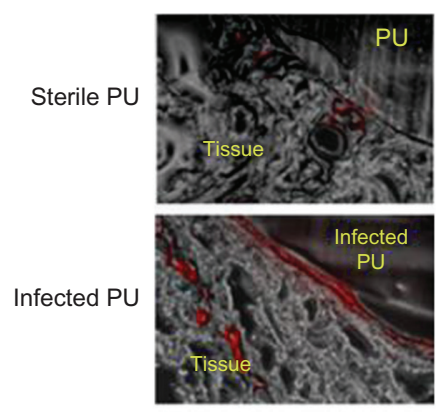

D

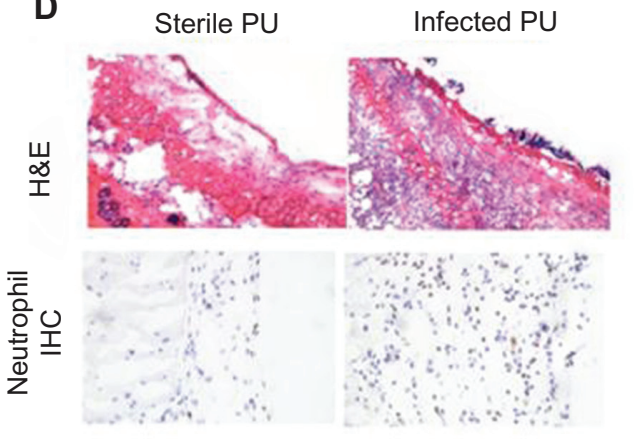

E

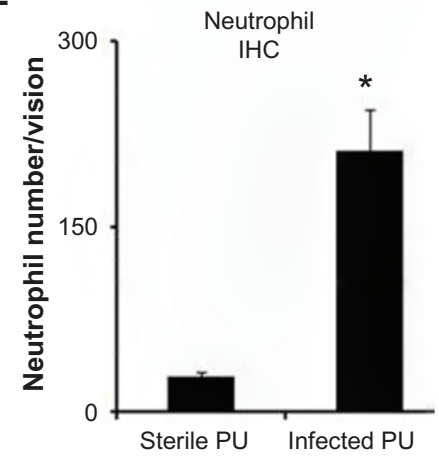

Figure 5 In an infected catheter inflammation model, polyurethane catheters were colonized with luciferase transgene Staphylococcus aureus and then transplanted subcutaneously on the back of animals for 24 hours. The animals were then administered with formyl peptide receptor-targeting nanoprobes for 3 hours prior to imaging analyses. (A) Representative bioluminescence image and the estimated bacterial colony-forming unit count depict bacterial colonization on an implanted catheter. (B) Fluorescence image and quantification of fluorescence intensity show formyl peptide receptor-targeting nanoprobe accumulation at the implantation site of infected polyurethane catheters. (C) Near-infrared fluorescence acquisition (red color) superimposed onto the correlative phase contrast microscopy image (20x) for tissues surrounding the polyurethane catheters. (D) Representative hematoxylin and eosin staining $(200 \times)$ and immunohistochemical staining of neutrophils (400x) and (E) neutrophil counts support the conclusion that substantially higher numbers of neutrophils were recruited to the infected polyurethane catheters than sterile ones.

Abbreviations: H\&E, hematoxylin and eosin; FPR, formyl peptide receptor; IHC, immunohistochemical; PU, polyurethane.

have shown that cFLFLF has a high binding affinity to the neutrophils' FPR. ${ }^{22,23}$ In addition, cFLFLF possesses antagonistic properties and does not induce neutropenia like other high-affinity chemotactic peptide analogs, such as formylmethionyl-leucyl-phenylalanine peptide. ${ }^{22,23}$ In the current study, a FPR-targeting (cFLFLF-conjugated) nanoprobe was fabricated using an eight-arm PEG core as a multifunctional platform. The eight-arm PEG core was chosen for several reasons. First, the multifunctional platform allowed optimization of the peptide density in a single nanoprobe, thus enhancing its targeting efficiency. ${ }^{26,40}$ Second, the modification of the peptide by PEGylation may enhance nanoprobe hydrophilicity, reduce nonspecific binding, and thus improve bioavailability and clearance when used in vivo. ${ }^{41,42}$ For in vivo imaging, Oyster-800 dye was chosen for its biocompatibility and high wavelength $(\sim 800 \mathrm{~nm})$ and because it has minimal tissue absorbance and background with improved fluorescence sensitivity. ${ }^{43,44}$

The in vitro experiments demonstrate that the FPRtargeting nanoprobe has high affinity and can specifically bind to neutrophils. These results concur with previous reports from microscopy studies of other fluorescentlylabeled chemotactic peptides..$^{2,45-47}$ By establishing a linear correlation between the fluorescence intensity and the neutrophil concentration, it was demonstrated that the nanoprobe may have some potential for quantitative analysis of neutrophils in vivo. ${ }^{25}$

Subcutaneous injection of LPS is a well-established inflammation model prompting severe inflammatory responses, accompanied by the recruitment of neutrophils, macrophages/monocytes, and other immune cells. ${ }^{48,49}$ Indeed, immediately following intravenous injection, FPR-targeting nanoprobes accumulated in the area of LPS-induced inflammation, which is consistent with previous findings that used a phorbol myristate acetate-induced acute inflammation model. ${ }^{23}$ Additionally, the substantial reduction of nanoprobe accumulation in neutrophil-depleted mice further supports the fact that the nanoprobe targets neutrophil recruitment and accumulation at the inflamed sites. Furthermore, the results show that FPR-targeting nanoprobes may also be used to detect mild inflammatory responses, such as foreign body reactions. 
The mechanism of FPR-targeting nanoprobe accumulation at the inflamed tissue is not entirely understood. The results suggest that the buildup of the FPR-targeting nanoprobe was likely caused by their interactions with FPR on the surfaces of activated neutrophils. This is supported by observations of the fluorescence intensity being reduced by approximately $82 \%$ following neutrophil depletion. The residual fluorescence intensity at the implantation site in neutrophil-depleted animals may be caused by the following reasons. First, neutrophil depletion can only reduce $\sim 80 \%$ of neutrophils from circulation, ${ }^{50}$ and a small number of residual neutrophils was still found to accumulate at the implant sites. Second, it is well established that biomaterial implants prompt histaminic reactions. ${ }^{51,52}$ Also, an "enhanced permeability and retention" effect at the implantation site due to leaky blood vessels may permit the permeation of a small amount of the imaging nanoprobes to the tissue surrounding implants. ${ }^{25,53}$

The ability of the FPR-targeting nanoprobe to detect and quantify foreign body reactions was tested using different materials. By implanting various polymeric particles, it has been confirmed that the FPR-targeting nanoprobe can be used to image and to assess the extent of neutrophil responses to different particle implants. Interestingly, optical imaging shows that the majority of the nanoprobes accumulate inside or in the tissue surrounding particle implants. Equally importantly, there is a good relationship between the extent of FPR-targeting nanoprobe fluorescence and the number of recruited neutrophils at the implant site. These results demonstrate that the FPR-targeting nanoprobes can be used not only to detect severe inflammation as reported earlier, ${ }^{19,22}$ but also to estimate the extent of the inflammatory response to biomaterials. Using an infected catheter implantation model, it was found that the bacteria-infected catheters significantly enhanced the localized accumulation of FPR-targeting nanoprobes in comparison to controls. Coincidentally, a large number of neutrophils were present nearby the infected catheter surface, which is in agreement with previous findings. ${ }^{54}$ Overall, the data supports the hypothesis that FPR-targeting nanoprobes may be used to detect neutrophil responses in the event of foreign body reactions and device-centered infection in vivo.

This study demonstrates that FPR-targeting nanoprobes can be fabricated to specifically detect activated neutrophils. Since neutrophils represent a major subset of inflammatory cells, and neutrophil interactions play an important role in the acute inflammatory response, it is anticipated that FPRtargeting nanoprobes may be a powerful tool to monitor the in vivo inflammatory response. Equally important, the FPR-targeting nanoprobe-based in vivo fluorescence imaging can provide an alternative method for analyzing a biomaterial's tissue compatibility in a rapid, noninvasive, and realtime manner. This would greatly improve the understanding of the processes and factors governing foreign body responses to biomaterials while reducing the number of animals needed to carry out extensive in vivo testing. It is worthwhile noting that the short tissue penetration depth of the fluorescence signal will limit the imaging modality's application in clinical practice. The inherent drawback, however, may be overcome through combinations of different imaging modalities such as positron emission tomography and magnetic resonance imaging in which the imaging nanoprobes could be conjugated to radionucleotides. Overall, the authors believe that the inflammatory cell-targeting imaging technique has a practical application in the evaluation and diagnosis of implant safety and performance.

\section{Conclusion}

Taking advantage of the high affinity of peptide cFLFLF to neutrophils' FPR, an FPR-targeting NIR nanoprobe was developed to detect activated neutrophils. The nanoprobes were found to have a high affinity and specificity for neutrophils in vitro as well as in vivo, with the ability to detect and quantify the degree of LPS-induced inflammatory responses and foreign body reactions. Further studies uncovered that the FPR-targeting nanoprobe can be used to distinguish sterile versus infected catheters. These results demonstrate that the FPR-targeting nanoprobe may be used as an effective, noninvasive, in vivo imaging tool for real-time evaluation of biomaterial safety and tissue compatibility.

\section{Acknowledgments}

This research is supported by National Institutes of Health grant EB007271 and American Heart Association Grant-in-Aid Award. The authors would also like to thank Dr Mohammad Omary and Dr Sreekar Marpu at University of North Texas (Denton, TX) for their facility support and technical assistance in conducting the fluorescence measurement.

\section{Disclosure}

The authors report no conflicts of interest in this work.

\section{References}

1. Anderson JM, Rodriguez A, Chang DT. Foreign body reaction to biomaterials. Semin Immunol. 2008;20(2):86-100.

2. Tang L, Eaton JW. Inflammatory responses to biomaterials. Am J Clin Pathol. 1995;103(4):466-471. 
3. Tang L, Eaton JW. Natural responses to unnatural materials: a molecular mechanism for foreign body reactions. Mol Med. 1999;5(6):351-358.

4. Tang L, Eaton JW. Fibrin(ogen) mediates acute inflammatory responses to biomaterials. J Exp Med. 1993;178(6):2147-2156.

5. Tang L, Lucas AH, Eaton JW. Inflammatory responses to implanted polymeric biomaterials: role of surface-adsorbed immunoglobulin G. J Lab Clin Med. 1993;122(3):292-300.

6. Kasahara Y, Iwai K, Yachie A, et al. Involvement of reactive oxygen intermediates in spontaneous and CD95 (Fas/APO-1)-mediated apoptosis of neutrophils. Blood. 1997;89(5):1748-1753.

7. Okusa MD. The inflammatory cascade in acute ischemic renal failure. Nephron. 2002;90(2):133-138.

8. Sutherland K, Mahoney JR II, Coury AJ, Eaton JW. Degradation of biomaterials by phagocyte-derived oxidants. J Clin Invest. 1993;92(5): 2360-2367.

9. Ye Q, Harmsen MC, van Luyn MJ, Bank RA. The relationship between collagen scaffold cross-linking agents and neutrophils in the foreign body reaction. Biomaterials. 2010;31(35):9192-9201.

10. Freeman TA, Parvizi J, Della Valle CJ. Steinbeck MJ. Reactive oxygen and nitrogen species induce protein and DNA modifications driving arthrofibrosis following total knee arthroplasty. Fibrogenesis Tissue Repair. 2009;2(1):5.

11. Hoemann CD, Chen G, Marchand C, et al. Scaffold-guided subchondral bone repair: implication of neutrophils and alternatively activated arginase-1+ macrophages. Am J Sports Med. 2010;38(9):1845-1856.

12. Kaplan SS, Heine RP, Simmons RL. Defensins impair phagocytic killing by neutrophils in biomaterial-related infection. Infect Immun. 1999;67(4):1640-1645.

13. Lefer AM, Campbell B, Scalia R, Lefer DJ. Synergism between platelets and neutrophils in provoking cardiac dysfunction after ischemia and reperfusion: role of selectins. Circulation. 1998;98(13):1322-1328.

14. Josefsson E, Tarkowski A, Carlsten H. Anti-inflammatory properties of estrogen. I. In vivo suppression of leukocyte production in bone marrow and redistribution of peripheral blood neutrophils. Cell Immunol. 1992;142(1):67-78.

15. Turner RF, Harrison DJ, Rajotte RV. Preliminary in vivo biocompatibility studies on perfluorosulphonic acid polymer membranes for biosensor applications. Biomaterials. 1991;12(4):361-368.

16. Schuh JC. Medical device regulations and testing for toxicologic pathologists. Toxicol Pathol. 2008;36(1):63-69.

17. Liu Z, Wyffels L, Barber C, Hui MM, Woolfenden JM. A (99m)Tclabeled dual-domain cytokine ligand for imaging of inflammation. $\mathrm{Nucl}$ Med Biol. 2011;38(6):795-805.

18. Kreisel D, Nava RG, Li W, et al. In vivo two-photon imaging reveals monocyte-dependent neutrophil extravasation during pulmonary inflammation. Proc Natl Acad Sci U S A. 2010;107(42):18073-18078.

19. Babich JW, Tompkins RG, Graham W, Barrow SA, Fischman AJ. Localization of radiolabeled chemotactic peptide at focal sites of Escherichia coli infection in rabbits: evidence for a receptor-specific mechanism. J Nucl Med. 1997;38(8):1316-1322.

20. van der Laken CJ, Boerman OC, Oyen WJ, et al. Technetium-99m-labeled chemotactic peptides in acute infection and sterile inflammation. $\mathrm{J} \mathrm{Nucl}$ Med. 1997;38(8):1310-1315.

21. Riou LM, Ruiz M, Sullivan GW, et al. Assessment of myocardial inflammation produced by experimental coronary occlusion and reperfusion with $99 \mathrm{mTc}-\mathrm{RP} 517$, a new leukotriene B4 receptor antagonist that preferentially labels neutrophils in vivo. Circulation. 2002;106(5):592-598.

22. Locke LW, Chordia MD, Zhang Y, et al. A novel neutrophil-specific PET imaging agent: cFLFLFK-PEG-64Cu. J Nucl Med. 2009;50(5): 790-797.

23. Xiao L, Zhang Y, Liu Z, Yang M, Pu L, Pan D. Synthesis of the Cyanine 7 labeled neutrophil-specific agents for noninvasive near infrared fluorescence imaging. Bioorg Med Chem Lett. 2010;20(12):3515-3517.

24. Zhang Y, Xiao L, Chordia MD, et al. Neutrophil targeting heterobivalent SPECT imaging probe: cFLFLF-PEG-TKPPR-99mTc. Bioconjug Chem. 2010;21(10):1788-1793.
25. Zhou J, Tsai YT, Weng H, Baker DW, Tang L. Real time monitoring of biomaterial-mediated inflammatory responses via macrophage-targeting NIR nanoprobes. Biomaterials. 2011;32(35):9383-9390.

26. Lifland AW, Zurla C, Santangelo PJ. Single molecule sensitive multivalent polyethylene glycol probes for RNA imaging. Bioconjug Chem. 2010;21:483-488.

27. Henderson RB, Hobbs JA, Mathies M, Hogg N. Rapid recruitment of inflammatory monocytes is independent of neutrophil migration. Blood. 2003;102(1):328-335

28. Weng $\mathrm{H}$, Zhou J, Tang L, Hu Z. Tissue responses to thermally-responsive hydrogel nanoparticles. J Biomater Sci Polym Ed. 2004;15(9): 1167-1180.

29. Zhou T, Wu WT, Zhou SQ. Engineering oligo(ethylene glycol)-based thermosensitive microgels for drug delivery applications. Polymer. 2010;51(17):3926-3933.

30. Hao Q, Chen Y, Zhu Y, et al. Neutrophil depletion decreases VEGFinduced focal angiogenesis in the mature mouse brain. J Cereb Blood Flow Metab. 2007;27(11):1853-1860.

31. Mermel LA, Stolz SM, Maki DG. Surface antimicrobial activity of heparin-bonded and antiseptic-impregnated vascular catheters. $J$ Infect Dis. 1993;167(4):920-924.

32. Kadurugamuwa JL, Modi K, Yu J, et al. Noninvasive biophotonic imaging for monitoring of catheter-associated urinary tract infections and therapy in mice. Infect Immun. 2005;73(7):3878-3887.

33. Lorenz U, Schafer T, Ohlsen K, et al. In vivo detection of Staphylococcus aureus in biofilm on vascular prostheses using non-invasive biophotonic imaging. Eur J Vasc Endovasc. 2011;41(1):68-75.

34. Baker DW, Liu X, Weng H, Luo C, Tang L. Fibroblast/fibrocyte: surface interaction dictates tissue reactions to micropillar implants. Biomacromolecules. 2011;12(4):997-1005.

35. Thevenot PT, Nair AM, Shen J, Lotfi P, Ko CY, Tang L. The effect of incorporation of SDF-1alpha into PLGA scaffolds on stem cell recruitment and the inflammatory response. Biomaterials. 2010;31(14): 3997-4008.

36. Knop K, Hoogenboom R, Fischer D, Schubert US. Poly(ethylene glycol) in drug delivery: pros and cons as well as potential alternatives. Angew Chem Int Ed Engl. 2010;49(36):6288-6308.

37. Cheng J, Teply BA, Sherifi I, et al. Formulation of functionalized PLGAPEG nanoparticles for in vivo targeted drug delivery. Biomaterials. 2007;28(5):869-876.

38. Hermanson GT. Bioconjugate Techniques. 2nd ed. San Diego: Academic Press; 2008.

39. Weng H, Zhou J, Tang L, Hu Z. Tissue responses to thermally-responsive hydrogel nanoparticles. J Biomater Sci Polym Ed. 2004;15(9): $1167-1180$

40. Fakhari A, Baoum A, Siahaan TJ, Le KB, Berkland C. Controlling ligand surface density optimizes nanoparticle binding to ICAM-1. J Pharm Sci. 2011;100(3):1045-1056.

41. Healy JM, Lewis SD, Kurz M, et al. Pharmacokinetics and biodistribution of novel aptamer compositions. Pharm Res. 2004;21(12): 2234-2246.

42. Suzuki T, Kanbara N, Tomono T, Hayashi N, Shinohara I. Physicochemical and biological properties of poly(ethylene glycol)coupled immunoglobulin G. Biochim Biophys Acta. 1984;788(2): 248-255.

43. Frangioni JV. In vivo near-infrared fluorescence imaging. Curr Opin Chem Biol. 2003;7(5):626-634.

44. Ntziachristos V, Bremer C, Weissleder R. Fluorescence imaging with near-infrared light: new technological advances that enable in vivo molecular imaging. Eur Radiol. 2003;13(1):195-208.

45. Niedel JE, Kahane I, Cuatrecasas P. Receptor-mediated internalization of fluorescent chemotactic peptide by human neutrophils. Science. 1979;205(4413):1412-1414.

46. Johansson B, Wymann MP, Holmgren-Peterson K, Magnusson KE. $\mathrm{N}$-formyl peptide receptors in human neutrophils display distinct membrane distribution and lateral mobility when labeled with agonist and antagonist. J Cell Biol. 1993;121(6):1281-1289. 
47. Stephenson KA, Banerjee SR, Besanger T, et al. Bridging the gap between in vitro and in vivo imaging: isostructural Re and $99 \mathrm{mTc}$ complexes for correlating fluorescence and radioimaging studies. $\mathrm{J} \mathrm{Am}$ Chem Soc. 2004;126(28):8598-8599.

48. Jeyaseelan S, Chu HW, Young SK, Worthen GS. Transcriptional profiling of lipopolysaccharide-induced acute lung injury. Infect Immun. 2004;72(12):7247-7256.

49. Zhou H, Andonegui G, Wong CH, Kubes P. Role of endothelial TLR4 for neutrophil recruitment into central nervous system microvessels in systemic inflammation. J Immunol. 2009;183(8):5244-5250.

50. Bhatia M, Saluja AK, Hofbauer B, Lee HS, Frossard JL, Steer ML. The effects of neutrophil depletion on a completely noninvasive model of acute pancreatitis-associated lung injury. Int J Pancreatol. 1998;24(2): $77-83$.
51. Zdolsek J, Eaton JW, Tang L. Histamine release and fibrinogen adsorption mediate acute inflammatory responses to biomaterial implants in humans. J Transl Med. 2007;5:31.

52. Tang L, Jennings TA, Eaton JW. Mast cells mediate acute inflammatory responses to implanted biomaterials. Proc Natl Acad Sci U S A. 1998;95(15):8841-8846.

53. Iyer AK, Khaled G, Fang J, Maeda H. Exploiting the enhanced permeability and retention effect for tumor targeting. Drug Discov Today. 2006;11(17-18):812-818.

54. Hirose K, Marui A, Arai Y, et al. A novel approach to reduce catheterrelated infection using sustained-release basic fibroblast growth factor for tissue regeneration in mice. Heart Vessels. 2007;22(4):261-267.

\section{Publish your work in this journal}

The International Journal of Nanomedicine is an international, peerreviewed journal focusing on the application of nanotechnology in diagnostics, therapeutics, and drug delivery systems throughout the biomedical field. This journal is indexed on PubMed Central, MedLine, CAS, SciSearch ${ }^{\circledR}$, Current Contents ${ }^{\circledR} /$ Clinical Medicine,
Journal Citation Reports/Science Edition, EMBase, Scopus and the Elsevier Bibliographic databases. The manuscript management system is completely online and includes a very quick and fair peer-review system, which is all easy to use. Visit http://www.dovepress.com/ testimonials.php to read real quotes from published authors. 